CERN-PH-TH/2004-132

\title{
Orientifolds, Brane Coordinates and Special Geometry $^{1}$
}

\author{
R. D’Auria *, S. Ferrara ${ }^{\dagger \ddagger}$ and M. Trigiante * \\ †CERN, Theory Division, CH 1211 Geneva 23, Switzerland. \\ $\ddagger I N F N$, Laboratori Nucleari di Frascati, Italy. \\ $\sharp$ University of California, Los Angeles, USA

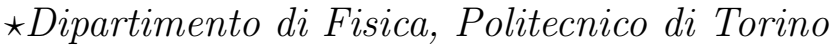 \\ C.so Duca degli Abruzzi, 24, I-10129 Torino, and \\ Istituto Nazionale di Fisica Nucleare, Sezione di Torino, \\ Italy
}

\begin{abstract}
We report on the gauged supergravity analysis of Type IIB vacua on $K 3 \times T^{2} / \mathbb{Z}_{2}$ orientifold in the presence of $D 3-D 7$-branes and fluxes. We discuss supersymmetric critical points correspond to Minkowski vacua and the related fixing of moduli, finding agreement with previous analysis. An important role is played by the choice of the symplectic holomorphic sections of special geometry which enter the computation of the scalar potential. The related period matrix $\mathscr{N}$ is explicitly given. The relation between the special geometry and the Born-Infeld action for the brane moduli is elucidated.
\end{abstract}

\footnotetext{
${ }^{1}$ To appear in the proceedings of "DeserFest" and "The status of M-theory" Ann Arbor, Michigan, 3-6 April 04.
} 


\section{Introduction}

We report on the four dimensional gauged-supergravity description of a class of $K 3 \times$ $T^{2} / \mathbb{Z}_{2}$ orientifolds [1, 2] in the presence of D3-D7 space-filling branes and three-form fluxes [3, 4].

In superstring and M-theory compactifications, which, in the absence of fluxes have an $N$-extended local supersymmetry, the low-energy dynamics is encoded in an effective supergravity theory with a certain number of matter multiplets, which describe the degrees of freedom of both bulk and brane excitations.

In particular in $\mathcal{N}=2$ supergravity in $D=4$ vector and hypermultiplets are described by special and quaternionic geometries, describing the moduli space of these theories.

When fluxes are turned on the effective supergravity theory undergoes a mass deformation which is encoded in a "gauged supergravity", whose general scalar potential is given in Sect. 3. This potential is completely fixed by the underlying scalar geometry, the

period matrix, the special geometry of the vector multiplets and by the Killing vectors of the gauged isometries of the quaternionic and special geometries.

A correct choice of the period matrix (explicitly given in Appendix B) and of the Killing isometries, would then allow to reproduce the the flux vacua with any number $\mathcal{N}=2,1,0$ of rigid supersymmetry in flat space [5]-[13. This is indeed the case and the agreement is found, for the particular choices of fluxes considered, with the compactification analysis of Tripathy and Trivedi [3].

These results are especially relevant because the supergravity effective potential can be further generalized to incorporate other perturbative or non-perturbative results [14] which may further stabilize the other moduli and lead to satisfactory inflationary cosmologies [15]-19].

From the point of view of the four-dimensional $\mathcal{N}=2$ effective supergravity, open string moduli, corresponding to D7 and D3-brane positions along $T^{2}$, form an enlargement of the vector multiplet moduli-space which is locally described, in absence of open-string moduli, by [4]:

$$
\left(\frac{\mathrm{SU}(1,1)}{\mathrm{U}(1)}\right)_{s} \times\left(\frac{\mathrm{SU}(1,1)}{\mathrm{U}(1)}\right)_{t} \times\left(\frac{\mathrm{SU}(1,1)}{\mathrm{U}(1)}\right)_{u}
$$

where $s, t, u$ denote the scalars of the vector multiplets containing the $K 3$-volume and the $\mathrm{R}-\mathrm{R}$ four-form on $K 3$, the $T^{2}$-complex structure, and the IIB axion-dilaton system, 
respectively $^{2}$ :

$$
\begin{aligned}
s & =C_{(4)}-\mathrm{i} \operatorname{Vol}\left(K_{3}\right), \\
t & =\frac{g_{12}}{g_{22}}-\mathrm{i} \frac{\sqrt{\operatorname{det} g}}{g_{22}}, \\
u & =C_{(0)}-\mathrm{i} e^{\varphi},
\end{aligned}
$$

where the matrix $g$ denotes the metric on $T^{2}$.

When D7-branes moduli are turned on, what is known is that $\mathrm{SU}(1,1)_{s}$ acts as an electric-magnetic duality transformation [20] both on the bulk and D7-brane vector fieldstrengths, while the $\mathrm{SU}(1,1)_{u}$ acts as an electric-magnetic duality transformation on the D3-vector field-strengths. Likewise the bulk vectors transform perturbatively under $\mathrm{SU}(1,1)_{u} \times \mathrm{SU}(1,1)_{t}$ while the D3-brane vectors do not transform under $\mathrm{SU}(1,1)_{s} \times$ $\mathrm{SU}(1,1)_{t}$ and the D7-brane vectors do not transform under $\mathrm{SU}(1,1)_{u} \times \mathrm{SU}(1,1)_{t}$.

All this is achieved starting from the following trilinear prepotential of special geometry 21]:

$$
\mathscr{F}\left(s, t, u, x^{k}, y^{r}\right)=s t u-\frac{1}{2} s x^{k} x^{k}-\frac{1}{2} u y^{r} y^{r},
$$

where $x^{k}$ and $y^{r}$ are the positions of the D7 and D3-branes along $T^{2}$ respectively, $k=$ $1, \ldots, n_{7}, r=1, \ldots, n_{3}$, and summation over repeated indices is understood. This prepotential is unique in order to preserve the shift-symmetries of the $s, t, u$ bulk complex fields up to terms which only depend on $x$ and $y$.

The above prepotential gives the correct answer if we set either all the $x^{k}$ or all the $y^{r}$ to zero. In this case the special geometry describes a symmetric space:

$$
\begin{aligned}
& \left(\frac{\mathrm{SU}(1,1)}{\mathrm{U}(1)}\right)_{s} \times \frac{\mathrm{SO}\left(2,2+n_{7}\right)}{\mathrm{SO}(2) \times \mathrm{SO}\left(2+n_{7}\right)}, \quad \text { for } y^{r}=0, \\
& \left(\frac{\mathrm{SU}(1,1)}{\mathrm{U}(1)}\right)_{u} \times \frac{\mathrm{SO}\left(2,2+n_{3}\right)}{\mathrm{SO}(2) \times \mathrm{SO}\left(2+n_{3}\right)}, \quad \text { for } x^{k}=0 .
\end{aligned}
$$

For both $x$ and $y$ non-vanishing, the complete Kähler manifold (of complex dimension $3+n_{3}+n_{7}$ ) is no longer a symmetric space even if it still has $3+n_{3}+n_{7}$ shift symmetries ${ }^{3}$.

Note that for $x^{k}=0$ the manifold is predicted as a truncation of the manifold describing the moduli-space of $T^{6} / \mathbb{Z}_{2} \mathcal{N}=4$ orientifold in the presence of D3-branes. The corresponding symplectic embedding was given in [23]. For $y^{r}=0$ the moduli-space is

\footnotetext{
${ }^{2}$ We notice that in 21] the imaginary parts of $u$ and $t$ were chosen to be positive. This however is inconsistent with the positivity domain of the vector kinetic terms which requires $s, t, u$ to have negative imaginary parts. Indeed $\operatorname{Im}(s)$ and $\operatorname{Im}(u)$ appear as coefficients in the kinetic terms of the $D 7$ and D3-brane vectors.

${ }^{3}$ The prepotential in eq. (3) actually corresponds to the homogeneous not symmetric spaces called $L\left(0, n_{7}, n_{3}\right)$ in 22 . We thank A. van Proeyen for a discussion on this point.
} 
predicted by the way $\mathrm{SU}(1,1)_{s}$ acts on both bulk and D7 vector fields. Upon compactification of Type IIB theory on $T^{2}$, the D7-brane moduli are insensitive to the further $K 3$ compactification and thus their gravity coupling must be the same as for vector multiplets coupled to supergravity in $D=8$. Indeed if $2+n$ vector multiplets are coupled to $\mathcal{N}=2$ supergravity in $D=8$, their non-linear $\sigma$-model is [24, 25]:

$$
\frac{\mathrm{SO}(2,2+n)}{\mathrm{SO}(2) \times \mathrm{SO}(2+n)} \times \mathbb{R}^{+} .
$$

Here $\mathbb{R}^{+}$denotes the volume of $T^{2}$ and the other part is the second factor in (4). Note that in $D=8, \mathcal{N}=2$ the $\mathrm{R}$-symmetry is $\mathrm{U}(1)$ which is the $\mathrm{U}(1)$ part of the $D=4, \mathcal{N}=2$ $\mathrm{U}(2) \mathrm{R}$-symmetry. The above considerations prove eq. (4).

Particular care is needed [26] when the effective supergravity is extended to include gauge couplings, as a result of turning on fluxes in the IIB compactification [27].

The reason is that the scalar potential depends explicitly on the symplectic embedding of the holomorphic sections of special geometry, while the Kähler potential, being symplectic invariant, does not. In fact, even in the analysis without open string moduli [4, it was crucial to consider a Calabi-Visentini basis where the $\mathrm{SO}(2,2)$ linearly acting symmetry on the bulk fields was $\mathrm{SU}(1,1)_{u} \times \mathrm{SU}(1,1)_{t}[28,22]$.

In the case at hand, the choice of symplectic basis is the one which corresponds to the Calabi-Visentini basis for $y^{r}=0$, with the $\mathrm{SU}(1,1)_{s}$ acting as an electric-magnetic duality transformation [4], but it is not such basis for the D3-branes even if the $x^{k}=0$. Indeed, for $x^{k}=0$, we must reproduce the mixed basis used for the $T^{6} / \mathbb{Z}_{2}$ orientifold [30, 31] in the presence of D3-branes found in [23. We note in this respect, that the choice of the symplectic section made in [19] does not determine type IIB vacua with the 3-form fluxes turned on. It does not correspond in fact to the symplectic embedding discussed in 44, 23] and [26]. The problem arises already in the absence of branes.

This report is organized as follows: in section 2 we review the gauged supergravity description of the model and briefly discuss the moduli stabilization of different vacua.

In section 3 we discuss the scalar potential in the presence of D3-D7 moduli.

In section 4 we consider the relation between the $\mathcal{N}=2$ special geometry corresponding to the D3-D7 system and the Born-Infeld action, taking into account the ChernSimons terms describing the couplings among bulk and brane moduli.

The final section is devoted to conclusions. Appendices A and B contain some relevant formulae for the scalar potential and the period matrix $\mathscr{N}_{\Lambda \Sigma}$ of the special geometry describing the bulk-brane coupled system of vector multiplets. 


\section{2. $\mathcal{N}=2$ and $\mathcal{N}=1$ supersymmetric cases.}

\section{1. $\mathcal{N}=2$ gauged supergravity}

We consider the gauging of $\mathcal{N}=2$ supergravity with a special geometry given by eq. (3). Let us briefly recall the main formulae of special Kähler geometry. The geometry of the manifold is encoded in the holomorphic section $\Omega=\left(X^{\Lambda}, F_{\Sigma}\right)$ which, in the special coordinate symplectic frame, is expressed in terms of a prepotential $\mathscr{F}\left(s, t, u, x^{k}, y^{r}\right)=$ $F\left(X^{\Lambda}\right) /\left(X^{0}\right)^{2}=\mathscr{F}\left(X^{\Lambda} / X^{0}\right)$, as follows:

$$
\Omega=\left(X^{\Lambda}, F_{\Lambda}=\partial F / \partial X^{\Lambda}\right)
$$

In our case $\mathscr{F}$ is given by eq. (3). The Kähler potential $K$ is given by the symplectic invariant expression:

$$
K=-\log \left[\mathrm{i}\left(\bar{X}^{\Lambda} F_{\Lambda}-\bar{F}_{\Lambda} X^{\Lambda}\right)\right]
$$

In terms of $K$ the metric has the form $g_{i \bar{\jmath}}=\partial_{i} \partial_{\bar{\jmath}} K$. The matrices $U^{\Lambda \Sigma}$ and $\overline{\mathscr{N}}_{\Lambda \Sigma}$ are respectively given by:

$$
\begin{aligned}
U^{\Lambda \Sigma} & =e^{K} \mathscr{D}_{i} X^{\Lambda} \mathscr{D}_{\bar{\jmath}} \bar{X}^{\Sigma} g^{i \bar{\jmath}}=-\frac{1}{2} \operatorname{Im}(\mathscr{N})^{-1}-e^{K} \bar{X}^{\Lambda} X^{\Sigma}, \\
\overline{\mathscr{N}}_{\Lambda \Sigma} & =\hat{h}_{\Lambda \mid I} \circ\left(\hat{f}^{-1}\right)^{I}{ }_{\Sigma}, \text { where } \hat{f}_{I}^{\Lambda}=\left(\begin{array}{c}
\mathscr{D}_{i} X^{\Lambda} \\
\bar{X}^{\Lambda}
\end{array}\right) ; \hat{h}_{\Lambda \mid I}=\left(\begin{array}{c}
\mathscr{D}_{i} F_{\Lambda} \\
\bar{F}_{\Lambda}
\end{array}\right) .
\end{aligned}
$$

For our choice of $\mathscr{F}, K$ has the following form:

$$
\begin{aligned}
K= & -\log \left[-8\left(\operatorname{Im}(s) \operatorname{Im}(t) \operatorname{Im}(u)-\frac{1}{2} \operatorname{Im}(s)\left(\operatorname{Im}(x)^{k}\right)^{2}-\right.\right. \\
& \left.\left.\frac{1}{2} \operatorname{Im}(u)\left(\operatorname{Im}(y)^{r}\right)^{2}\right)\right]
\end{aligned}
$$

with $\operatorname{Im}(s), \operatorname{Im}(t), \operatorname{Im}(u)<0$ at $x^{k}=y^{r}=0$. The components $X^{\Lambda}, F_{\Sigma}$ of the symplectic section which correctly describe our problem, are chosen by performing a constant symplectic change of basis from the one in (7) given in terms of the prepotential in eq. (3). The symplectic matrix is

$$
\left(\begin{array}{cc}
A & -B \\
B & A
\end{array}\right)
$$


The rotated symplectic sections then become

$$
\begin{aligned}
X^{0} & =\frac{1}{\sqrt{2}}\left(1-t u+\frac{\left(x^{k}\right)^{2}}{2}\right), \quad X^{1}=-\frac{t+u}{\sqrt{2}}, \\
X^{2} & =-\frac{1}{\sqrt{2}}\left(1+t u-\frac{\left(x^{k}\right)^{2}}{2}\right), \quad X^{3}=\frac{t-u}{\sqrt{2}}, \\
X^{k} & =x^{k}, \quad X^{r}=y^{r}, \\
F_{0} & =\frac{s\left(2-2 t u+\left(x^{k}\right)^{2}\right)+u\left(y^{r}\right)^{2}}{2 \sqrt{2}}, \quad F_{1}=\frac{-2 s(t+u)+\left(y^{r}\right)^{2}}{2 \sqrt{2}} \\
F_{2} & =\frac{s\left(2+2 t u-\left(x^{k}\right)^{2}\right)-u\left(y^{r}\right)^{2}}{2 \sqrt{2}}, \quad F_{3}=\frac{2 s(-t+u)+\left(y^{r}\right)^{2}}{2 \sqrt{2}} \\
F_{i} & =-s x^{k}, \quad F_{r}=-u y^{r} .
\end{aligned}
$$

Note that, since $\partial X^{\Lambda} / \partial s=0$ the new sections do not admit a prepotential, and the no-go theorem on partial supersymmetry breaking 32 does not apply in this case. As in [4, we limit ourselves to gauge shift-symmetries of the quaternionic manifold of the K3 moduli-space. Other gaugings which include the gauge group on the brane will be considered elsewhere.

We will also consider particular assignments of the gauge couplings which give $X^{2}=$ $X^{3}=0$, i.e. $t=u=-i$. Other choices for the gauge couplings, allowing $u \neq-i$ are possible and we shall discuss some cases here.

\section{2. $\mathcal{N}=2$ supersymmetric critical points}

In the sequel we limit our analysis to critical points in flat space. The $\mathcal{N}=2$ critical points demand $\mathscr{P}_{\Lambda}^{x}=0$. This equation does not depend on the special geometry and its solution is the same as in [4], i.e. $g_{2}, g_{3} \neq 0, g_{0}=g_{1}=0$ and $e_{a}^{m}=0$ for $a=1$, 2, were the Killing vectors gauged by the fields $A_{\mu}^{2}$ and $A_{\mu}^{3}$ are constants and their non-vanishing components are $k_{2}^{u}=g_{2}$ along the direction $q^{u}=C^{a=1}$ and $k_{3}^{u}=g_{3}$ along the direction

$q^{u}=C^{a=2}$. The 22 fields $C^{m}, C^{a}, m=1,2,3$ and $a=1, \ldots, 19$ denote the Peccei-Quinn scalars. The vanishing of the hyperino-variation further demands:

$$
k_{\Lambda}^{u} X^{\Lambda}=0 \Rightarrow X^{2}=X^{3}=0 \Leftrightarrow t=u, 1+t^{2}=\frac{\left(x^{k}\right)^{2}}{2} .
$$

Hence for $\mathcal{N}=2$ vacua the D7 and D3-brane positions are still moduli while the axiondilaton and $T^{2}$ complex structure are stabilised.

\section{3. $\mathcal{N}=1,0$ critical points}

The $\mathcal{N}=1$ critical points in flat space studied in [4] were first obtained by setting $g_{0}, g_{1} \neq 0$ and $g_{2}=g_{3}=0$, with $k_{0}^{u}=g_{0}$ along the direction $q^{u}=C^{m=1}$ and $k_{1}^{u}=g_{1}$ along the direction $q^{u}=C^{m=2}$. 
Constant Killing spinors. By imposing $\delta_{\epsilon_{2}} f=0$ for the variations of the fermionic fields $f$ we get the following:

From the hyperino variations:

$$
\begin{gathered}
\delta_{\epsilon_{2}} \zeta^{A a}=0 \Rightarrow e_{m}^{a}=0 m=1,2 ; a=1, \ldots, 19 \\
\delta_{\epsilon_{2}} \zeta^{A}=0 \Rightarrow \text { vanishing of the gravitino variation } .
\end{gathered}
$$

The gravitino variation vanishes if:

$$
S_{22}=-g_{0} X^{0}+\mathrm{i} g_{1} X^{1}=0 .
$$

From the gaugino variations we obtain:

$$
\delta_{\epsilon_{2}}\left(\lambda^{\bar{\imath}}\right)_{A}=0 \Rightarrow e^{\frac{K}{2}} \mathscr{P}_{\Lambda}^{x}\left(\partial_{i} X^{\Lambda}+\left(\partial_{i} K\right) X^{\Lambda}\right) \sigma_{A 2}^{x}=0
$$

the second term (with $\partial_{i} K$ ) gives a contribution proportional to the gravitino variation while the first term, for $i=u, t, x^{k}$ respectively gives:

$$
\begin{aligned}
-g_{0} \partial_{u} X^{0}+\mathrm{i} g_{1} \partial_{u} X^{1} & =0 \\
-g_{0} \partial_{t} X^{0}+\mathrm{i} g_{1} \partial_{t} X^{1} & =0 \\
-g_{0} \partial_{x^{k}} X^{0} & =0
\end{aligned}
$$

for $i=y^{r}$ the equation is identically satisfied. From the last equation we get $x^{k}=0$ and the other two, together with $S_{22}=0$ give $u=t=-\mathrm{i}, g_{0}=g_{1}$.

So we see that for $\mathcal{N}=1$ vacua the D7-brane coordinates are frozen while the D3brane coordinates remain moduli. This agrees with the analysis of [3]. If $g_{0} \neq g_{1}$ the above solutions give critical points with vanishing cosmological constant but with no supersymmetry left.

More general $\mathcal{N}=1,0$ vacua can be obtained also in this case by setting $g_{2}, g_{3} \neq 0$. The only extra conditions coming from the gaugino variations for $\mathcal{N}=1$ vacua is that $e_{m}^{a=1,2}=0$. This eliminates from the spectrum two extra metric scalars $e_{3}^{a=1,2}$ and the $C_{a=1,2}$ axions. These critical points preserve $\mathcal{N}=1$ or not depending on whether $\left|g_{0}\right|=$ $\left|g_{1}\right|$ or not.

We can describe the $\mathcal{N}=1 \rightarrow \mathcal{N}=0$ transition with an $\mathcal{N}=1$ no-scale supergravity [33, 34] based on a constant superpotential and a non-linear sigma-model which is

$$
\frac{\mathrm{U}\left(1,1+n_{3}\right)}{\mathrm{U}(1) \times \mathrm{U}\left(1+n_{3}\right)} \times \frac{\mathrm{SO}(2,18)}{\mathrm{SO}(2) \times \mathrm{SO}(18)},
$$

where the two factors come from vector multiplets and hypermultiplets, respectively. This model has vanishing scalar potential, reflecting the fact that there are not further scalars 
becoming massive in this transition [4]. We further note that any superpotential $W(y)$ for the D3 brane coordinates would generate a potential [35] term

$$
e^{K} K^{y \bar{y}} \partial_{y} W \partial_{\bar{y}} \bar{W}
$$

which then would require the extra condition $\partial_{y} W=0$ for a critical point with vanishing vacuum energy.

The residual moduli space of K3 metrics at fixed volume is locally given by

$$
\frac{\mathrm{SO}(1,17)}{\mathrm{SO}(17)}
$$

We again remark that we have considered vacua with vanishing vacuum energy. We do not consider here the possibility of other vacua with non-zero vacuum energy, as i.e. in 19].

\subsection{More general vacua}

There are more general critical points defined by values of $t, u$ different form $-i$ and depending on ratios of fluxes. Let us give an instance of this for the $\mathcal{N}=2$ preserving vacua.

Consider the situation with generic flux $f^{p}{ }_{\Lambda}, p=(m, a), \Lambda=0, \ldots, 3$, which corresponds to the charge-couplings:

$$
\nabla_{\mu} C^{p}=\partial_{\mu} C^{p}+f^{p}{ }_{\Lambda} A_{\mu}^{\Lambda}
$$

For a $\mathcal{N}=2$ vacuum, for the vanishing of the gravitino and gaugino variations, we need $\mathscr{P}_{\Lambda}^{x}=0$, where

$$
\mathscr{P}_{\Lambda}^{x}=\omega_{u}^{x} k_{\Lambda}^{u} \equiv \omega_{p}^{x} f_{\Lambda}^{p}
$$

From the hyperino variations we have

$$
k_{\Lambda}^{u} X^{\Lambda}=f_{\Lambda}^{p} X^{\Lambda}=0
$$

We take $\Lambda=2,3$ with $f_{2,3}^{p} \neq 0$ for $p=a,(a=1 \ldots, 19)$ and $f_{\Lambda}^{p}=0$ otherwise. The hyperino variation then is:

$$
f_{2}^{a} X^{2}+f^{a}{ }_{3} X^{3}=0
$$

Setting $f_{2}^{a}=\alpha f^{a}{ }_{3}$ we obtain

$$
f^{a}{ }_{3}\left(\alpha X^{2}+X^{3}\right)=0
$$

that is

$$
\frac{X^{3}}{X^{2}}=\frac{u-t}{1+t u-\frac{\left(x^{k}\right)^{2}}{2}}=-\alpha=-\frac{f_{2}^{a_{2}}}{f^{a_{3}}}
$$


The condition $\mathscr{P}_{\Lambda}^{x}=0$ on the other hand implies

$$
e^{x} f_{2,3}^{a}=0,
$$

but since $f^{a}{ }_{2}=\alpha f^{a}{ }_{3}$ then the above equation is equivalent to the following single condition

$$
e^{x} f_{2}^{a}=0
$$

namely it fixes only one triplet of metric moduli.

This vacuum preserves $\mathcal{N}=2$ supersymmetry with one massive vector multiplet corresponding to a combination of $A_{\mu}^{2}$ and $A_{\mu}^{3}$. Moreover condition (26) fixes the $T^{2}$ complex structure modulus in terms of the axion-dilaton and the $x^{k}$ moduli of the D7 brane coordinates. Note that in the previous solution 21] $X^{2}=X^{3}=0, u=t, t^{2}=$ $-1+\frac{\left(x^{k}\right)^{2}}{2}$ and $x^{k}$ were still unfixed. For $\alpha=0$ or $\infty$ we get $X^{3}$ or $X^{2}$ vanishing which corresponds to the example given in 3 .

\section{The potential}

The general form of the $\mathcal{N}=2$ scalar potential is:

$$
V=4 e^{K} h_{u v} k_{\Lambda}^{u} k_{\Sigma}^{v} X^{\Lambda} \bar{X}^{\Sigma}+e^{K} g_{i \bar{\jmath}} k_{\Lambda}^{i} k_{\Sigma}^{\bar{\jmath}} X^{\Lambda} \bar{X}^{\Sigma}+e^{K}\left(U^{\Lambda \Sigma}-3 e^{K} X^{\Lambda} \bar{X}^{\Sigma}\right) \mathscr{P}_{\Lambda}^{x} \mathscr{P}_{\Sigma}^{x},
$$

where the second term is vanishing for abelian gaugings. Here $h_{u v}$ is the quaternionic metric and $k_{\Lambda}^{u}$ the quaternionic Killing vector of the hypermultiplet $\sigma$-model.

The scalar potential, at the extremum of the $e_{m}^{a}$ scalars, has the following form ${ }^{4}$ :

$$
\begin{aligned}
V= & 4 e^{2 \phi} e^{K}\left[\sum_{\Lambda=0}^{3}\left(g_{\Lambda}\right)^{2}\left|X^{\Lambda}\right|^{2}+\frac{1}{2}\left(g_{0}^{2}+g_{1}^{2}\right)(t-\bar{t})\left((u-\bar{u})-\frac{1}{2} \frac{\left(x^{k}-\bar{x}^{k}\right)^{2}}{(t-\bar{t})}\right)\right. \\
& \left.+\frac{\left(y^{r}-\bar{y}^{r}\right)^{2}}{8(s-\bar{s})(u-\bar{u})}\left(g_{0}^{2}\left(\bar{u} x^{k}-\bar{x}^{k} u\right)^{2}+g_{1}^{2}\left(x^{k}-\bar{x}^{k}\right)^{2}\right)\right] .
\end{aligned}
$$

From the above expression we see that in the $\mathcal{N}=2$ case, namely for $g_{0}=g_{1}=0$, the potential depends on $y^{r}$ only through the factor $e^{K}$ and vanishes identically in $y^{r}$ for the values of the $t, u$ scalars given in (13), for which $X^{2}=X^{3}=0$. If $g_{0}$ or $g_{1}$ are nonvanishing $(\mathcal{N}=1,0$ cases $)$ the extremisation of the potential with respect to $x^{k}$, namely $\partial_{x^{k}} V=0$ fixes $x^{k}=0$. For $x^{k}=0$ the potential depends on $y^{r}$ only through the factor $e^{K}$ and vanishes identically in $y^{r}$ for $t=u=-\mathrm{i}$.

\footnotetext{
${ }^{4}$ Note that there is a misprint in eq. (5.1) of ref. 4]. The term $e^{2 \phi} e^{\tilde{K}} g_{0} g_{1}\left(X_{0} \bar{X}_{1}+X_{1} \bar{X}_{0}\right)$ is actually absent
} 


\section{Special coordinates, solvable coordinates and B.I. action}

The prepotential for the spacial geometry of the $D 3-D 7$ system, given in (3), was obtained in 21], by using arguments based on duality symmetry, four dimensional ChernSimons terms coming from the p-brane couplings as well as couplings of vector multiplets in $D=4$ and $D=8$.

A similar result was advocated in [36, 37] by performing first a $K 3$ reduction to $D=6$ and then further compactifying the theory to $D=4$ on $T^{2}$.

The subtlety of this derivation is that the naive Born-Infeld action derived for D5 and $D 9$ branes in $D=6$ gives kinetic terms for the scalar fields which, at the classical level, are inconsistent with $\mathcal{N}=2$ supersymmetry. This is a consequence of the fact that anomalies are present in the theory, as in the $D=10$ case. The mixed anomaly local counterterms are advocated to make the Lagrangian $\mathcal{N}=2$ supersymmetric in $D=4$.

Therefore the corrected Lagrangian, in the original brane coordinates is highly nonpolinomial. In fact the original Born-Infeld, Chern-Simons naive (additive) classical scalar action

$$
\begin{aligned}
& \frac{\left|\partial s^{\prime}+c^{r} \partial d^{r}\right|^{2}}{\left(s^{\prime}-\bar{s}^{\prime}\right)^{2}}+\frac{\left|\partial u^{\prime}+a^{i} \partial b^{i}\right|^{2}}{\left(u^{\prime}-\bar{u}^{\prime}\right)^{2}}+\frac{\left|t^{\prime} \partial d^{r}+\partial c^{r}\right|^{2}}{\left(s^{\prime}-\bar{s}^{\prime}\right)\left(t^{\prime}-\bar{t}^{\prime}\right)}+\frac{\left|t^{\prime} \partial b^{i}+\partial a^{i}\right|^{2}}{\left(u^{\prime}-\bar{u}^{\prime}\right)\left(t^{\prime}-\bar{t}^{\prime}\right)}+\frac{\left|\partial t^{\prime}\right|^{2}}{\left(t^{\prime}-\bar{t}^{\prime}\right)^{2}} \\
& s^{\prime}=s-\frac{1}{2} d^{r} y^{r} ; u^{\prime}=u-\frac{1}{2} b^{i} x^{i} ; t^{\prime}=t \\
& x^{i}=a^{i}+t b^{i} ; y^{r}=c^{r}+t d^{r}
\end{aligned}
$$

has a metric which was shown [37] to be Kähler with Kähler potential ${ }^{5}$

$$
\begin{aligned}
K= & -\log \left[(s-\bar{s})(t-\bar{t})-\frac{1}{2}\left(y^{r}-\bar{y}^{r}\right)^{2}\right]-\log \left[(u-\bar{u})(t-\bar{t})-\frac{1}{2}\left(x^{i}-\bar{x}^{i}\right)^{2}\right]+\log (t-\bar{t}) \\
& =-\log Y_{S K}-\log \left(1+\frac{X_{4}}{Y_{S G}}\right)
\end{aligned}
$$

where

$$
\begin{aligned}
X_{4} & =\frac{\left(x^{i}-\bar{x}^{i}\right)^{2}\left(y^{r}-\bar{y}^{r}\right)^{2}}{4(t-\bar{t})} \\
Y_{S K} & =(s-\bar{s})(t-\bar{t})(u-\bar{u})-\frac{1}{2}(u-\bar{u})\left(y^{r}-\bar{y}^{r}\right)^{2}-\frac{1}{2}(s-\bar{s})\left(x^{i}-\bar{x}^{i}\right)^{2},
\end{aligned}
$$

where here and in the following summation over repeated indices is understood. Therefore the correction to the scalar metric in the brane coordinates is:

$$
\partial_{p} \partial_{\bar{q}} \Delta K=\partial_{p} \partial_{\bar{q}} \log \left(1+\frac{X_{4}}{Y_{S G}}\right) .
$$

\footnotetext{
${ }^{5} Y_{S K}$ differs by a factor $-i$ from the special geometry formula obtained from the prepotential in 3
} 
It is clear that the classical brane coordinates are not good "supersymmetric" coordinates, in that the corrected action is not polynomial in them. From the fact that the combined system is a homogeneous space, we indeed expect that suitable coordinates exist such that the quantum corrected $(\mathcal{N}=2$ supersymmetric) action has a simple polynomial dependence on them, including the interference term. Such coordinates do indeed exist and allow to write the combined Born-Infeld action and supersymmetric counterterms, in a manifest supersymmetric way. Modulo field redefinitions, these coordinates reduce to the standard brane coordinates when either the $D 3$ or the $D 7$-branes are absent, in which cases the homogeneous space becomes a symmetric space. This parametrization in terms of "supersymmetric" coordinates, corresponds to the solvable Lie algebra description of the manifold first introduced by Alekseevski 38, 39, which we shall discuss in what follows. In Alekseevski's notation the manifold under consideration is of type $K\left(n_{3}, n_{7}\right)$ which can be written as:

$$
\begin{aligned}
K\left(n_{3}, n_{7}\right) & =W\left(g_{\alpha}, h_{\alpha}, Y^{ \pm}, Z^{ \pm}\right) \\
\operatorname{dim}\left(Y^{ \pm}\right) & =n_{3} ; \operatorname{dim}\left(Z^{ \pm}\right)=n_{7},
\end{aligned}
$$

where $n_{3}$ and $n_{7}$ denote the number of $D 3$ and $D 7$-branes respectively. Our identification of the scalar fields with solvable parameters is described by the following expression for a generic solvable Lie algebra element:

$$
\begin{gathered}
\text { Solv }=\left\{\sum_{\alpha=t, u, s} \varphi^{\alpha} h_{\alpha}+\hat{\theta}_{t} g_{t}+\theta_{u} g_{u}+\theta_{s} g_{s}+y^{r \pm} Y_{r}^{ \pm}+z^{i \pm} Z_{i}^{ \pm}\right\} \\
\hat{\theta}_{t}=\theta_{t}+y^{r+} y^{r-}+z^{i+} z^{i-}
\end{gathered}
$$

where $\left(y^{r+}, y^{r-}\right)$ and $\left(z^{i+}, z^{i-}\right)$ are related to the real and imaginary parts of the $D 3$ and D7-branes complex coordinates along $T^{2}$. The non trivial commutation relations between the above solvable generators are:

$$
\begin{aligned}
{\left[h_{t}, Y^{ \pm}\right] } & =\frac{1}{2} Y^{ \pm} ; \quad\left[h_{t}, Z^{ \pm}\right]=\frac{1}{2} Z^{ \pm} \\
{\left[h_{s}, Y^{ \pm}\right] } & = \pm \frac{1}{2} Y^{ \pm} ; \quad\left[h_{u}, Z^{ \pm}\right]= \pm \frac{1}{2} Z^{ \pm} \\
{\left[g_{s}, Y^{-}\right] } & =Y^{+} ; \quad\left[g_{u}, Z^{-}\right]=Z^{+}, \\
{\left[Y_{r}^{+}, Y_{s}^{-}\right] } & =\delta_{r s} g_{t} ; \quad\left[Z_{i}^{+}, Z_{j}^{-}\right]=\delta_{i j} g_{t} ; r, s=1, \ldots, n_{3} i, j=1, \ldots, n_{7}, \\
{\left[h_{\alpha}, g_{\alpha}\right] } & =g_{\alpha} ; \alpha=t, u, s .
\end{aligned}
$$

We exponentiate the solvable algebra using the following coset-representative:

$$
L=e^{\theta_{s} g_{s}} e^{y^{r-} Y_{r}^{-}} e^{y^{r+} Y_{r}^{+}} e^{\theta_{u} g_{u}} e^{z^{i-} Z_{i}^{-}} e^{z^{i+} Z_{i}^{+}} e^{\hat{\theta}_{t} g_{t}} e^{\varphi^{\alpha} h_{\alpha}} .
$$

The order of the exponentials in the coset representative and the particular parameter $\hat{\theta}_{t}$ used for $g_{t}$, have been chosen in such a way that the axions $\theta_{s}, \theta_{t}, \theta_{u}, y^{r+}, z^{i+}$ appear in 
the resulting metric only covered by derivatives. The metric reads:

$$
\begin{aligned}
d s^{2}= & \left(d \varphi_{\alpha}\right)^{2}+e^{-2 \varphi_{t}}\left(d \theta_{t}+\frac{1}{2} d \theta_{u}\left(z^{-}\right)^{2}+\frac{1}{2} d \theta_{s}\left(y^{-}\right)^{2}+z^{i-} d z^{i+}+y^{r-} d y^{r+}\right)^{2}+ \\
& e^{-2 \varphi_{u}} d \theta_{u}^{2}+e^{-2 \varphi_{s}} d \theta_{s}^{2}+e^{-\varphi_{t}-\varphi_{u}}\left(d z^{i+}+d \theta_{u} z^{i-}\right)^{2}+e^{-\varphi_{t}+\varphi_{u}}\left(d z^{i-}\right)^{2}+ \\
& e^{-\varphi_{t}-\varphi_{s}}\left(d y^{r+}+d \theta_{s} y^{r-}\right)^{2}+e^{-\varphi_{t}+\varphi_{s}}\left(d y^{r-}\right)^{2} \\
& \left(z^{+}\right)^{2} \equiv \sum_{i=1}^{n_{7}}\left(z^{i+}\right)^{2} ;\left(y^{+}\right)^{2} \equiv \sum_{r=1}^{n_{3}}\left(y^{r+}\right)^{2}
\end{aligned}
$$

Identifying the axionic coordinates $\theta_{s}, \theta_{t}, \theta_{u}, y^{r+}, z^{i+}$ with the real part of the special coordinates $s, t, u, y^{r}, x^{i}$, and comparing the corresponding components of the metric one easily obtains the following relations between the solvable coordinates and the special coordinates:

$$
\begin{aligned}
s & =\theta_{s}-\frac{i}{2} e^{\varphi_{s}} ; u=\theta_{u}-\frac{i}{2} e^{\varphi_{u}}, \\
t & =\theta_{t}-\frac{i}{2}\left(e^{\varphi_{t}}+\frac{1}{2} e^{\varphi_{u}}\left(z^{-}\right)^{2}+\frac{1}{2} e^{\varphi_{s}}\left(y^{-}\right)^{2}\right), \\
x^{i} & =z^{i+}+\frac{i}{2} e^{\varphi_{u}} z^{i-} ; y^{r}=y^{r+}+\frac{i}{2} e^{\varphi_{s}} y^{r-} .
\end{aligned}
$$

Note that the classical B-I+C-S action (31), with no interference term in the D3 $(c, d)$ and $D 7(a, b)$ brane coordinates is still described by a homogeneous manifold spanned by the following $2 n_{3}+2 n_{7}+6$ isometries:

$$
\begin{aligned}
u & \rightarrow e^{\lambda_{u}} u ; \delta u=u_{0}+a_{0}^{i} b^{i}, \\
s & \rightarrow e^{\lambda_{s}} s ; \delta s=s_{0}+c_{0}^{r} d_{r}, \\
t & \rightarrow e^{\lambda_{t}} t ; \delta t=t_{0}, \\
c^{r} & \rightarrow e^{\frac{\lambda_{s}+\lambda_{t}}{2}} c^{r} ; \delta c^{r}=t_{0} d^{r}, \\
d^{r} & \rightarrow e^{\frac{\lambda_{s}-\lambda_{t}}{2}} d^{r} ; \delta d^{r}=d_{0}^{r}, \\
a^{i} & \rightarrow e^{\frac{\lambda_{u}+\lambda_{t}}{2}} a^{i} ; \delta a^{i}=a_{0}^{i}+t_{0} b^{i}, \\
b^{i} & \rightarrow e^{\frac{\lambda_{u}-\lambda_{t}}{2}} b^{i} ; \delta b^{i}=b_{0}^{i} .
\end{aligned}
$$

The underlying homogeneous space is generated by the following rank 3 solvable Lie algebra $\left\{T_{a}^{i}, T_{b}^{i}, T_{c}^{r}, T_{d}^{r}, h_{s}, h_{t}, h_{u}, g_{s}, g_{t}, g_{u}\right\}$ whose non trivial commutation relations are:

$$
\begin{aligned}
{\left[T_{a}^{i}, T_{b}^{j}\right] } & =\delta^{i j} g_{u} ; \quad\left[T_{c}^{r}, T_{d}^{s}\right]=\delta^{r s} g_{s} \\
{\left[T_{b}^{i}, g_{t}\right] } & =T_{a}^{i} ; \quad\left[T_{d}^{r}, g_{t}\right]=T_{c}^{r} \\
{\left[h_{\alpha}, g_{\alpha}\right] } & =g_{\alpha} \quad \alpha=s, t, u \\
{\left[h_{s}, T_{d}^{r}\right] } & =\frac{1}{2} T_{d}^{r} ; \quad\left[h_{s}, T_{c}^{r}\right]=\frac{1}{2} T_{c}^{r}
\end{aligned}
$$




$$
\begin{aligned}
{\left[h_{u}, T_{b}^{i}\right] } & =\frac{1}{2} T_{b}^{i} ; \quad\left[h_{u}, T_{a}^{i}\right]=\frac{1}{2} T_{a}^{i} \\
{\left[h_{t}, T_{d}^{r}\right] } & =-\frac{1}{2} T_{d}^{r} ;\left[h_{t}, T_{c}^{r}\right]=\frac{1}{2} T_{c}^{r} \\
{\left[h_{t}, T_{b}^{i}\right] } & =-\frac{1}{2} T_{b}^{i} ; \quad\left[h_{t}, T_{a}^{i}\right]=\frac{1}{2} T_{a}^{i},
\end{aligned}
$$

where the nilpotent generators have been labelled by the corresponding axionic scalar fields. This space is not a subspace of the original quanternionic space, but it becomes so if we set either $a, b=0$ and exchange the role of $s$ and $t$ or if we set $c, d=0$ and exchange the role of $u$ and $t$.

The amazing story is that the coordinates in $D=4$ corresponding to the supersymmetric theory, deform this space into an other homogeneous space generated by the isometries in (37) which corresponds to an $\mathcal{N}=2$ special geometry.

The relation between the solvable Lie algebra generators $\left\{T_{a}^{i}, T_{b}^{i}, T_{c}^{r}, T_{d}^{r}, h_{s}, h_{t}, h_{u}\right.$ $\left., g_{s}, g_{t}, g_{u}\right\}$ corresponding to the classical coordinates and the solvable generators $\left\{Y^{ \pm}, Z^{ \pm}, h_{\alpha}, g_{\alpha}\right\}$ corresponding to the "supersymmetric" coordinates is the following:

$$
\begin{aligned}
& T_{a}^{i}=\hat{Z}^{i+} ; T_{b}^{i}=\hat{Z}^{i-}, \\
& T_{c}^{r}=\hat{Y}^{r+} ; T_{d}^{r}=\hat{Y}^{r-}
\end{aligned}
$$

where $\hat{Y}$ and $\hat{Z}$ are the generators with opposite grading with respect to $Y$ and $Z$ respectively. It can be shown that in the manifold $K\left(n_{3}, n_{7}\right), \hat{Y}$ or $\hat{Z}$ are isometries only if $n_{7}=0$ or $n_{3}=0$ respectively. Indeed in these two cases the manifold is symmetric and each solvable nilpotent isometry has a "hidden" counterpart with opposite grading. Otherwise the manifold spanned by the classical coordinates and the manifold parametrized by the "supersymmetric" ones are in general different.

\section{Conclusions}

The present investigation allows us to study in a fairly general way the potential for the 3-form flux compactification, in presence of both bulk and open string moduli. In absence of fluxes the D3, D7 dependence of the Kähler potential is rather different since this moduli couple in different ways to the bulk moduli.

Moreover, in the presence of 3 -form fluxes which break $\mathcal{N}=2 \rightarrow \mathcal{N}=1,0$ the D7 moduli are stabilised while the D3 moduli are not. For small values of the coordinates $x^{k}, y^{r}$ the dependence of their kinetic term is (for $\left.u=t=-\mathrm{i}\right),-\left(\partial_{\mu} \bar{y}^{r} \partial^{\mu} y^{r}\right) / \operatorname{Im}(s)$ for the D3-brane moduli, and $-\left(\partial_{\mu} \bar{x}^{k} \partial^{\mu} x^{k}\right)$ for the D7-brane moduli. This is in accordance with the suggestion of [15]. Note that the above formulae, at $x=0, u=t=-\mathrm{i}$ are

true up to corrections $O\left(\frac{\operatorname{Im}(y)^{2}}{\operatorname{Im}(s)}\right)$, since $y$ and $s$ are moduli even in presence of fluxes. The 
actual dependence of these terms on the compactification volume is important in order to further consider models for inflatons where the terms in the scalar potential allow to stabilise the remaining moduli.

Finally, we have not considered here the gauging of compact gauge groups which exist on the brane world-volumes. This is, for instance, required [40, 41, 19] in models with hybrid inflation [42. This issue will be considered elsewhere.

Acknowledgements. Work supported in part by the European Community's Human Potential Program under contract HPRN-CT-2000-00131 Quantum Space-Time, in which R. D'A. is associated to Torino University. The work of S.F. has been supported in part by European Community's Human Potential Program under contract HPRN-CT-2000-00131

Quantum Space-Time, in association with INFN Frascati National Laboratories and by D.O.E. grant DE-FG03-91ER40662, Task C.

\section{Appendix A Some relevant formulae.}

We are interested in gauging the 22 translations in the coset $\mathrm{SO}(4,20) /(\mathrm{SO}(3,19) \times$ $\mathrm{O}(1,1))$. Let us denote by $L$ the coset representative of $\mathrm{SO}(3,19) / \mathrm{SO}(3) \times \mathrm{SO}(19)$. It will be written in the form:

$$
L=\left(\begin{array}{cc}
\left(1+\mathbf{e ~}^{T}\right)^{\frac{1}{2}} & -\mathbf{e} \\
-\mathbf{e}^{T} & \left(1+\mathbf{e}^{T} \mathbf{e}\right)^{\frac{1}{2}}
\end{array}\right),
$$

where $\mathbf{e}=\left\{e^{m}{ }_{a}\right\}, \mathbf{e}^{T}=\left\{e^{a}{ }_{m}\right\}, m=1,2,3$ and $a=1, \ldots, 19$, are the coordinates of the manifold. The 22 nilpotent Peccei-Quinn generators are denoted by $\left\{Z_{m}, Z_{a}\right\}$ and the gauge generators are:

$$
t_{\Lambda}=f^{m}{ }_{\Lambda} Z_{m}+h^{a}{ }_{\Lambda} Z_{a},
$$

the corresponding Killing vectors have non vanishing components: $k_{\Lambda}^{m}=f^{m}{ }_{\Lambda}$ and $k_{\Lambda}^{a}=$ $h^{a}{ }_{\Lambda}$. The moment maps are:

$$
\mathscr{P}_{\Lambda}^{x}=\sqrt{2}\left(e^{\phi}\left(L^{-1}\right)^{x}{ }_{m} f^{m}{ }_{\Lambda}+e^{\phi}\left(L^{-1}\right)^{x}{ }_{a} h^{a}{ }_{\Lambda}\right),
$$

where $\phi$ is the $T^{2}$ volume modulus 4$]: e^{-2 \phi}=\operatorname{Vol}\left(T^{2}\right)$ and $x=1,2,3$. The metric along the Peccei-Quinn directions $I=(m, a)$ is:

$$
h_{I J}=e^{2 \phi}\left(\delta_{I J}+2 e^{a}{ }_{I} e^{a}{ }_{J}\right) .
$$

The potential has the following form:

$$
V=4 e^{2 \phi}\left(f^{m}{ }_{\Lambda} f^{m}{ }_{\Sigma}+2 e^{a}{ }_{m} e^{a}{ }_{n} f^{m}{ }_{\Lambda} f^{n}{ }_{\Sigma}+h^{a}{ }_{\Lambda} h^{a}{ }_{\Sigma}\right) \bar{L}^{\Lambda} L^{\Sigma}
$$




$$
\begin{aligned}
& +2 e^{2 \phi}\left(U^{\Lambda \Sigma}-3 \bar{L}^{\Lambda} L^{\Sigma}\right)\left(f^{m}{ }_{\Lambda} f^{m}{ }_{\Sigma}+e^{a}{ }_{m} e^{a}{ }_{n} f^{m}{ }_{\Lambda} f^{n}{ }_{\Sigma}\right. \\
& \left.\left.+2\left[\left(1+\mathbf{e e}^{T}\right)^{\frac{1}{2}}\right]_{m}^{n} e^{n}{ }_{a} f^{m}{ }_{(\Lambda} h^{a}{ }_{\Sigma}\right)+e^{n}{ }_{a} e^{n}{ }_{b} h^{a}{ }_{\Lambda} h^{b}{ }_{\Sigma}\right) .
\end{aligned}
$$

In all the models we consider, at the extremum point of the potential in the special Kähler manifold the following condition holds: $\left(U^{\Lambda \Sigma}-3 \bar{L}^{\Lambda} L^{\Sigma}\right)_{\mid 0} f^{m}{ }_{(\Lambda} h^{a}{ }_{\Sigma)}=0$. As a consequence of this, as it is clear from (5), the potential in this point depends on the metric scalars $e_{a}^{m}$ only through quadratic terms in the combinations $e^{m}{ }_{a} h^{a}{ }_{\Lambda}$ and $e^{a}{ }_{m} f^{m}{ }_{\Lambda}$. Therefore $V$ is extremised with respect to the $e_{a}^{m}$ scalars once we restrict ourselves to the moduli defined as follows:

$$
\text { moduli: } \quad e^{m}{ }_{a} h^{a}{ }_{\Lambda}=e^{a}{ }_{m} f^{m}{ }_{\Lambda}=0 .
$$

The vanishing of the potential implies

$$
\left(U^{\Lambda \Sigma}-\bar{L}^{\Lambda} L^{\Sigma}\right)_{\mid 0} f_{(\Lambda}^{m} f^{m}{ }_{\Sigma)}+2\left(\bar{L}^{\Lambda} L^{\Sigma}\right)_{\mid 0} h_{(\Lambda}^{a} h_{\Sigma)}^{a}=0 .
$$

Furthermore, one may notice that, as in [4, the following relations hold in all the models under consideration:

$$
\left(U^{\Lambda \Sigma}-\bar{L}^{\Lambda} L^{\Sigma}\right)_{\mid 0} f^{m}{ }_{(\Lambda} f^{m}{ }_{\Sigma)}=\left(\bar{L}^{\Lambda} L^{\Sigma}\right)_{\mid 0} h_{(\Lambda}^{a} h^{a}{ }_{\Sigma)}=0 .
$$

Our analysis is limited to the case in which the only non-vanishing $f$ and $h$ constants are:

$$
\begin{aligned}
f^{1}{ }_{0} & =g_{0} ; f^{2}{ }_{1}=g_{1} ; h^{1}{ }_{2}=g_{2} ; h^{2}{ }_{3}=g_{3} ; h^{2+k}{ }_{3+k}=g_{4}^{k} \\
h_{3+n_{7}+r}^{2+n_{7}+r} & =g_{5}^{r} .
\end{aligned}
$$

\section{Appendix B The matrix $\mathscr{N}$.}

Using the special geometry formula (9) it is possible to compute the matrix $\mathscr{N}_{\Lambda \Sigma}$ for any choice of the symplectic section, including those cases for which no prepotential exists. For the sake of simplicity we will suppress the indices $k$ and $r$ in $x^{k}$ and $y^{r}$ by considering the case $n_{3}=n_{7}=1$. Moreover we will express the complex coordinates in terms of their real and imaginary parts:

$$
s=s_{1}+\mathrm{i} s_{2} ; t=t_{1}+\mathrm{i} t_{2} ; u=u_{1}+\mathrm{i} u_{2} ; x=x_{1}+\mathrm{i} x_{2} ; y=y_{1}+\mathrm{i} y_{2}
$$

Let the $D 7$ and $D 3$ brane vectors correspond to the values $\Lambda=4,5$ respectively. We list below the independent components of the real and imaginary parts of $\mathscr{N}$ :

$$
\operatorname{Re}(\mathscr{N})_{0,0}=s_{1}-\frac{1}{2} u_{1} y_{1}^{2}+\frac{u_{2}\left(-2+2 t_{1} u_{1}-x_{1}^{2}\right) y_{1} y_{2}}{2 t_{2} u_{2}-x_{2}^{2}}-
$$




$$
\begin{aligned}
& \frac{\left(-1+t_{1} u_{1}-\frac{1}{2} x_{1}^{2}\right)\left(2 t_{1} u_{2}^{2}+x_{2}\left(-2 u_{2} x_{1}+u_{1} x_{2}\right)\right) y_{2}^{2}}{\left(-2 t_{2} u_{2}+x_{2}^{2}\right)^{2}} \\
& \operatorname{Re}(\mathscr{N})_{0,1}=\frac{y_{1}\left(-2 t_{2} u_{2} y_{1}+x_{2}^{2} y_{1}+4\left(t_{1}+u_{1}\right) u_{2} y_{2}\right)}{8 t_{2} u_{2}-4 x_{2}^{2}}+ \\
& \frac{y_{2}^{2}\left(2 u_{2}^{2}\left(2-2 t_{1}\left(t_{1}+2 u_{1}\right)+x_{1}^{2}\right)+4\left(t_{1}+u_{1}\right) u_{2} x_{1} x_{2}\right)}{4\left(-2 t_{2} u_{2}+x_{2}^{2}\right)^{2}}+ \\
& \frac{\left(2-2 u_{1}\left(2 t_{1}+u_{1}\right)+x_{1}^{2}\right) x_{2}^{2} y_{2}^{2}}{4\left(-2 t_{2} u_{2}+x_{2}^{2}\right)^{2}} \\
& \operatorname{Re}(\mathscr{N})_{0,2}=\frac{1}{2} u_{1} y_{1}^{2}+\frac{u_{2}\left(2 t_{1} u_{1}-x_{1}^{2}\right) y_{1} y_{2}}{-2 t_{2} u_{2}+x_{2}^{2}}+ \\
& \frac{\left(t_{1} u_{1}-\frac{1}{2} x_{1}^{2}\right)\left(2 t_{1} u_{2}^{2}+x_{2}\left(-2 u_{2} x_{1}+u_{1} x_{2}\right)\right) y_{2}^{2}}{\left(-2 t_{2} u_{2}+x_{2}^{2}\right)^{2}} \\
& \operatorname{Re}(\mathscr{N})_{0,3}=\frac{y_{1}\left(-2 t_{2} u_{2} y_{1}+x_{2}^{2} y_{1}+4\left(t_{1}-u_{1}\right) u_{2} y_{2}\right)}{8 t_{2} u_{2}-4 x_{2}^{2}}+ \\
& \frac{-\left(u_{2}\left(u_{2}\left(2+2 t_{1}^{2}-4 t_{1} u_{1}+x_{1}^{2}\right)+2\left(-t_{1}+u_{1}\right) x_{1} x_{2}\right) y_{2}^{2}\right)}{2\left(-2 t_{2} u_{2}+x_{2}^{2}\right)^{2}}+ \\
& \frac{\left(2+2 u_{1}\left(-2 t_{1}+u_{1}\right)+x_{1}^{2}\right) x_{2}^{2} y_{2}^{2}}{4\left(-2 t_{2} u_{2}+x_{2}^{2}\right)^{2}} \\
& \operatorname{Re}(\mathscr{N})_{0,4}=-\frac{x_{1} y_{2}\left(-4 t_{2} u_{2}^{2} y_{1}+2 t_{1} u_{2}^{2} y_{2}+u_{1} x_{2}^{2} y_{2}\right)}{\sqrt{2}\left(-2 t_{2} u_{2}+x_{2}^{2}\right)^{2}}+ \\
& \frac{u_{2} x_{2} y_{2}\left(-2 x_{1} x_{2} y_{1}+\left(2-2 t_{1} u_{1}+3 x_{1}^{2}\right) y_{2}\right)}{\sqrt{2}\left(-2 t_{2} u_{2}+x_{2}^{2}\right)^{2}} \\
& \operatorname{Re}(\mathscr{N})_{0,5}=\frac{2 t_{2} u_{1} u_{2} y_{1}-u_{1} x_{2}^{2} y_{1}+u_{2}\left(2-2 t_{1} u_{1}+x_{1}^{2}\right) y_{2}}{\sqrt{2}\left(2 t_{2} u_{2}-x_{2}^{2}\right)} \\
& \operatorname{Re}(\mathscr{N})_{1,1}=s_{1}-\frac{\left(t_{1}+u_{1}\right)\left(2 u_{2}^{2}+x_{2}^{2}\right) y_{2}^{2}}{\left(-2 t_{2} u_{2}+x_{2}^{2}\right)^{2}} \\
& \operatorname{Re}(\mathscr{N})_{1,2}=\frac{y_{1}\left(2 t_{2} u_{2} y_{1}-x_{2}^{2} y_{1}-4\left(t_{1}+u_{1}\right) u_{2} y_{2}\right)}{8 t_{2} u_{2}-4 x_{2}^{2}}+ \\
& \frac{u_{2}\left(u_{2}\left(2+2 t_{1}^{2}+4 t_{1} u_{1}-x_{1}^{2}\right)-2\left(t_{1}+u_{1}\right) x_{1} x_{2}\right) y_{2}^{2}}{2\left(-2 t_{2} u_{2}+x_{2}^{2}\right)^{2}}+ \\
& \frac{\left(2+2 u_{1}\left(2 t_{1}+u_{1}\right)-x_{1}^{2}\right) x_{2}^{2} y_{2}^{2}}{4\left(-2 t_{2} u_{2}+x_{2}^{2}\right)^{2}} \\
& \operatorname{Re}(\mathscr{N})_{1,3}=\frac{\left(2 u_{1} u_{2}^{2}-t_{1} x_{2}^{2}\right) y_{2}^{2}}{\left(-2 t_{2} u_{2}+x_{2}^{2}\right)^{2}} \\
& \operatorname{Re}(\mathscr{N})_{1,4}=-\frac{\left(2 u_{2}^{2} x_{1}+2\left(t_{1}+u_{1}\right) u_{2} x_{2}+x_{1} x_{2}^{2}\right) y_{2}^{2}}{\sqrt{2}\left(-2 t_{2} u_{2}+x_{2}^{2}\right)^{2}} \\
& \operatorname{Re}(\mathscr{N})_{1,5}=\frac{\sqrt{2}\left(2 t_{2} u_{2} y_{1}-x_{2}^{2} y_{1}-2\left(t_{1}+u_{1}\right) u_{2} y_{2}\right)}{4 t_{2} u_{2}-2 x_{2}^{2}} \\
& \operatorname{Re}(\mathscr{N})_{2,2}=-s_{1}-\frac{1}{2} u_{1} y_{1}^{2}+\frac{u_{2}\left(2+2 t_{1} u_{1}-x_{1}^{2}\right) y_{1} y_{2}}{2 t_{2} u_{2}-x_{2}^{2}}-
\end{aligned}
$$




$$
\begin{aligned}
& \frac{\left(1+t_{1} u_{1}-\frac{1}{2} x_{1}^{2}\right)\left(2 t_{1} u_{2}^{2}+x_{2}\left(-2 u_{2} x_{1}+u_{1} x_{2}\right)\right) y_{2}^{2}}{\left(-2 t_{2} u_{2}+x_{2}^{2}\right)^{2}} \\
& \operatorname{Re}(\mathscr{N})_{2,3}=\frac{y_{1}\left(2 t_{2} u_{2} y_{1}-x_{2}^{2} y_{1}+4\left(-t_{1}+u_{1}\right) u_{2} y_{2}\right)}{8 t_{2} u_{2}-4 x_{2}^{2}}+ \\
& \frac{u_{2}\left(u_{2}\left(-2+2 t_{1}^{2}-4 t_{1} u_{1}+x_{1}^{2}\right)+2\left(-t_{1}+u_{1}\right) x_{1} x_{2}\right) y_{2}^{2}}{2\left(-2 t_{2} u_{2}+x_{2}^{2}\right)^{2}}+ \\
& \frac{\left(2+4 t_{1} u_{1}-2 u_{1}^{2}-x_{1}^{2}\right) x_{2}^{2} y_{2}^{2}}{4\left(-2 t_{2} u_{2}+x_{2}^{2}\right)^{2}} \\
& \operatorname{Re}(\mathscr{N})_{2,4}=\frac{x_{1} y_{2}\left(-4 t_{2} u_{2}^{2} y_{1}+2 t_{1} u_{2}^{2} y_{2}+u_{1} x_{2}^{2} y_{2}\right)}{\sqrt{2}\left(-2 t_{2} u_{2}+x_{2}^{2}\right)^{2}}+ \\
& \frac{u_{2} x_{2} y_{2}\left(2 x_{1} x_{2} y_{1}-3 x_{1}^{2} y_{2}+2\left(y_{2}+t_{1} u_{1} y_{2}\right)\right)}{\sqrt{2}\left(-2 t_{2} u_{2}+x_{2}^{2}\right)^{2}} \\
& \operatorname{Re}(\mathscr{N})_{2,5}=\frac{-2 t_{2} u_{1} u_{2} y_{1}+u_{1} x_{2}^{2} y_{1}+u_{2}\left(2+2 t_{1} u_{1}-x_{1}^{2}\right) y_{2}}{\sqrt{2}\left(2 t_{2} u_{2}-x_{2}^{2}\right)} \\
& \operatorname{Re}(\mathscr{N})_{3,3}=-s_{1}+\frac{\left(t_{1}-u_{1}\right)\left(2 u_{2}^{2}-x_{2}^{2}\right) y_{2}^{2}}{\left(-2 t_{2} u_{2}+x_{2}^{2}\right)^{2}} \\
& \operatorname{Re}(\mathscr{N})_{3,4}=\frac{\left(2 u_{2}^{2} x_{1}+2\left(-t_{1}+u_{1}\right) u_{2} x_{2}-x_{1} x_{2}^{2}\right) y_{2}^{2}}{\sqrt{2}\left(-2 t_{2} u_{2}+x_{2}^{2}\right)^{2}} \\
& \operatorname{Re}(\mathscr{N})_{3,5}=\frac{\sqrt{2}\left(2 t_{2} u_{2} y_{1}-x_{2}{ }^{2} y_{1}+2\left(-t_{1}+u_{1}\right) u_{2} y_{2}\right)}{4 t_{2} u_{2}-2 x_{2}{ }^{2}} \\
& \operatorname{Re}(\mathscr{N})_{4,4}=-s_{1}-\frac{4 u_{2} x_{1} x_{2} y_{2}^{2}}{\left(-2 t_{2} u_{2}+x_{2}^{2}\right)^{2}} \\
& \operatorname{Re}(\mathscr{N})_{4,5}=\frac{2 u_{2} x_{1} y_{2}}{-2 t_{2} u_{2}+x_{2}{ }^{2}} \\
& \operatorname{Re}(\mathscr{N})_{5,5}=-u_{1}
\end{aligned}
$$

As far as $\operatorname{Im}(\mathscr{N})$ is concerned, its independent entries are:

$$
\begin{aligned}
\operatorname{Im}(\mathscr{N})_{0,0}= & \frac{s_{2}\left(4+4\left(t_{1}{ }^{2}+t_{2}{ }^{2}\right)\left(u_{1}{ }^{2}+u_{2}{ }^{2}\right)+x_{1}{ }^{4}+x_{2}{ }^{4}+2 x_{1}{ }^{2}\left(2+x_{2}{ }^{2}\right)\right)}{8 t_{2} u_{2}-4 x_{2}{ }^{2}}+ \\
& \frac{s_{2} t_{2}\left(-2 u_{1} x_{1} x_{2}+u_{2}\left(x_{1}-x_{2}\right)\left(x_{1}+x_{2}\right)\right)}{2 t_{2} u_{2}-x_{2}{ }^{2}}+ \\
& -\frac{s_{2} t_{1}\left(2 u_{2} x_{1} x_{2}+u_{1}\left(2+x_{1}{ }^{2}-x_{2}{ }^{2}\right)\right)}{2 t_{2} u_{2}-x_{2}{ }^{2}}+ \\
& \frac{y_{1}}{2}\left(u_{2} y_{1}+\frac{2\left(2 t_{1} u_{2}{ }^{2}+x_{2}\left(-2 u_{2} x_{1}+u_{1} x_{2}\right)\right) y_{2}}{-2 t_{2} u_{2}+x_{2}{ }^{2}}\right)+ \\
& \frac{-u_{2}\left(4 t_{1}{ }^{2}\left(u_{1}{ }^{2}-u_{2}{ }^{2}\right)+4 t_{2}{ }^{2}\left(u_{1}{ }^{2}+u_{2}{ }^{2}\right)-4 t_{1} u_{1}\left(2+x_{1}{ }^{2}\right)+\left(2+x_{1}\right)^{2}\right) y_{2}{ }^{2}}{4\left(-2 t_{2} u_{2}+x_{2}\right)^{2}{ }^{2}}+ \\
& \frac{x_{2}\left(t_{2} u_{1}{ }^{2} x_{2}+u_{2}\left(-2 t_{1} u_{2} x_{1}+\left(t_{1} u_{1}+t_{2} u_{2}+x_{1}{ }^{2}\right) x_{2}\right)\right) y_{2}{ }^{2}}{\left(-2 t_{2} u_{2}+x_{2}{ }^{2}\right)^{2}}+ \\
& \frac{-x_{2}{ }^{3}\left(4 u_{1} x_{1}+u_{2} x_{2}\right) y_{2}{ }^{2}}{4\left(-2 t_{2} u_{2}+x_{2}{ }^{2}\right)^{2}} \\
\operatorname{Im}(\mathscr{N})_{0,1}= & \frac{s_{2}\left(-2\left(t_{2}+u_{2}\right) x_{1} x_{2}+u_{1}\left(-2+2 t_{1}{ }^{2}+2 t_{2}{ }^{2}-x_{1}{ }^{2}+x_{2}{ }^{2}\right)\right)}{4 t_{2} u_{2}-2 x_{2}{ }^{2}}+ \\
& \frac{s_{2} t_{1}\left(-2+2 u_{1}{ }^{2}+2 u_{2}{ }^{2}-x_{1}{ }^{2}+x_{2}{ }^{2}\right)}{4 t_{2} u_{2}-2 x_{2}{ }^{2}}+
\end{aligned}
$$




$$
\begin{aligned}
& -\frac{y_{2}\left(t_{2}^{2} u_{1} u_{2} y_{2}-t_{1} u_{2}^{3} y_{2}+t_{2}\left(2 u_{2}^{3} y_{1}+u_{2} x_{2}^{2} y_{1}-u_{1} x_{2}^{2} y_{2}\right)\right)}{\left(-2 t_{2} u_{2}+x_{2}^{2}\right)^{2}}+ \\
& \frac{-\left(\left(t_{1}+u_{1}\right) u_{2}\left(-2+2 t_{1} u_{1}-x_{1}^{2}-x_{2}^{2}\right) y_{2}^{2}\right)}{2\left(-2 t_{2} u_{2}+x_{2}^{2}\right)^{2}}+ \\
& \frac{x_{2}\left(2 u_{2}^{2}+x_{2}^{2}\right) y_{2}\left(x_{2} y_{1}-x_{1} y_{2}\right)}{2\left(-2 t_{2} u_{2}+x_{2}^{2}\right)^{2}} \\
& \operatorname{Im}(\mathscr{N})_{0,2}=-\frac{s_{2}\left(-4+4 t_{1}{ }^{2}\left(u_{1}^{2}+u_{2}^{2}\right)+4 t_{2}{ }^{2}\left(u_{1}^{2}+u_{2}^{2}\right)+x_{1}^{4}+2 x_{1}^{2} x_{2}^{2}+x_{2}{ }^{4}\right)}{8 t_{2} u_{2}-4 x_{2}{ }^{2}}+ \\
& \frac{s_{2}\left(t_{1}\left(2 u_{2} x_{1} x_{2}+u_{1}\left(x_{1}^{2}-x_{2}^{2}\right)\right)+t_{2}\left(2 u_{1} x_{1} x_{2}+u_{2}\left(-x_{1}^{2}+x_{2}^{2}\right)\right)\right)}{2 t_{2} u_{2}-x_{2}^{2}}+ \\
& \frac{y_{1}\left(-2 t_{2} u_{2}^{2} y_{1}+4 t_{1} u_{2}^{2} y_{2}+2 u_{1} x_{2}^{2} y_{2}+u_{2} x_{2}\left(x_{2} y_{1}-4 x_{1} y_{2}\right)\right)}{4 t_{2} u_{2}-2 x_{2}^{2}}+ \\
& \frac{-\left(u_{2}\left(4-4\left(t_{1}^{2}+t_{2}^{2}\right) u_{1}^{2}+4\left(t_{1}-t_{2}\right)\left(t_{1}+t_{2}\right) u_{2}^{2}+4 t_{1} u_{1} x_{1}^{2}-x_{1}^{4}\right) y_{2}^{2}\right)}{4\left(-2 t_{2} u_{2}+x_{2}^{2}\right)^{2}}+ \\
& -\frac{x_{2}\left(\left(t_{2}\left(u_{1}^{2}+u_{2}^{2}\right)+u_{2} x_{1}^{2}\right) x_{2}+t_{1} u_{2}\left(-2 u_{2} x_{1}+u_{1} x_{2}\right)\right) y_{2}^{2}}{\left(-2 t_{2} u_{2}+x_{2}^{2}\right)^{2}}+ \\
& \frac{x_{2}^{3}\left(4 u_{1} x_{1}+u_{2} x_{2}\right) y_{2}^{2}}{4\left(-2 t_{2} u_{2}+x_{2}^{2}\right)^{2}} \\
& \operatorname{Im}(\mathscr{N})_{0,3}=\frac{s_{2}\left(2\left(-t_{2}+u_{2}\right) x_{1} x_{2}+u_{1}\left(2+2 t_{1}^{2}+2 t_{2}^{2}+x_{1}^{2}-x_{2}^{2}\right)\right)}{4 t_{2} u_{2}-2 x_{2}{ }^{2}}+ \\
& \frac{-\left(s_{2} t_{1}\left(2+2 u_{1}^{2}+2 u_{2}^{2}+x_{1}^{2}-x_{2}^{2}\right)\right)+\left(2 u_{2}^{2}-x_{2}^{2}\right) y_{1} y_{2}}{4 t_{2} u_{2}-2 x_{2}^{2}}+ \\
& \frac{-\left(u_{1}\left(-2 t_{2} x_{2}^{2}+u_{2}\left(2+2 t_{1}^{2}+2 t_{2}^{2}+x_{1}^{2}+x_{2}^{2}\right)\right) y_{2}^{2}\right)}{2\left(-2 t_{2} u_{2}+x_{2}^{2}\right)^{2}}+ \\
& \frac{-\left(\left(x_{1} x_{2}\left(-2 u_{2}^{2}+x_{2}^{2}\right)-t_{1} u_{2}\left(2+2 u_{1}^{2}-2 u_{2}^{2}+x_{1}^{2}+x_{2}^{2}\right)\right) y_{2}^{2}\right)}{2\left(-2 t_{2} u_{2}+x_{2}^{2}\right)^{2}}
\end{aligned}
$$

$$
\begin{aligned}
& \operatorname{Im}(\mathscr{N})_{0,4}=-\frac{s_{2}\left(x_{1}^{3}-2\left(t_{2} u_{1}+t_{1} u_{2}\right) x_{2}+x_{1}\left(2-2 t_{1} u_{1}+2 t_{2} u_{2}+x_{2}^{2}\right)\right)}{\sqrt{2}\left(2 t_{2} u_{2}-x_{2}^{2}\right)}+ \\
& \frac{y_{2}\left(-4 t_{2} u_{2}^{2} x_{2} y_{1}+2 u_{2} x_{2}{ }^{3} y_{1}+2 t_{1} u_{2}^{2} x_{2} y_{2}+u_{1} x_{2}{ }^{3} y_{2}\right)}{\sqrt{2}\left(-2 t_{2} u_{2}+x_{2}^{2}\right)^{2}}+ \\
& \frac{u_{2} x_{1}\left(2-2 t_{1} u_{1}+x_{1}^{2}-2 x_{2}^{2}\right) y_{2}^{2}}{\sqrt{2}\left(-2 t_{2} u_{2}+x_{2}^{2}\right)^{2}} \\
& \sqrt{2}\left(-2 t_{2} u_{2}+x_{2}^{2}\right)^{2} \\
& \operatorname{Im}(\mathscr{N})_{0,5}=\frac{\sqrt{2}\left(-2 t_{2} u_{2}^{2} y_{1}+2 t_{1} u_{2}^{2} y_{2}+u_{1} x_{2}^{2} y_{2}+u_{2} x_{2}\left(x_{2} y_{1}-2 x_{1} y_{2}\right)\right)}{4 t_{2} u_{2}-2 x_{2}^{2}} \\
& \operatorname{Im}(\mathscr{N})_{1,1}=\frac{s_{2}\left(t_{1}^{2}+t_{2}^{2}+2 t_{1} u_{1}+u_{1}^{2}+u_{2}^{2}+x_{2}^{2}\right)}{2 t_{2} u_{2}-x_{2}^{2}}+ \\
& \frac{\left(\left(-t_{2}^{2}-\left(t_{1}+u_{1}\right)^{2}\right) u_{2}+u_{2}^{3}+\left(t_{2}+u_{2}\right) x_{2}^{2}\right) y_{2}^{2}}{\left(-2 t_{2} u_{2}+x_{2}^{2}\right)^{2}} \\
& \operatorname{Im}(\mathscr{N})_{1,2}=-\frac{s_{2}\left(-2\left(t_{2}+u_{2}\right) x_{1} x_{2}+u_{1}\left(2+2 t_{1}^{2}+2 t_{2}^{2}-x_{1}^{2}+x_{2}^{2}\right)\right)}{4 t_{2} u_{2}-2 x_{2}^{2}}+ \\
& -\frac{s_{2} t_{1}\left(2+2 u_{1}^{2}+2 u_{2}^{2}-x_{1}^{2}+x_{2}^{2}\right)}{4 t_{2} u_{2}-2 x_{2}{ }^{2}}+ \\
& \frac{y_{2}\left(t_{2}{ }^{2} u_{1} u_{2} y_{2}-t_{1} u_{2}{ }^{3} y_{2}+t_{2}\left(2 u_{2}^{3} y_{1}+u_{2} x_{2}{ }^{2} y_{1}-u_{1} x_{2}^{2} y_{2}\right)\right)}{\left(-2 t_{2} u_{2}+x_{2}{ }^{2}\right)^{2}}+ \\
& \frac{-\left(u_{2} y_{2}\left(\left(-t_{1}-u_{1}\right)\left(2+2 t_{1} u_{1}-x_{1}^{2}-x_{2}^{2}\right) y_{2}+2 u_{2} x_{2}\left(x_{2} y_{1}-x_{1} y_{2}\right)\right)\right)}{2\left(-2 t_{2} u_{2}+x_{2}^{2}\right)^{2}}+ \\
& \frac{-\left(x_{2}^{3} y_{2}\left(x_{2} y_{1}-x_{1} y_{2}\right)\right)}{2\left(-2 t_{2} u_{2}+x_{2}^{2}\right)^{2}} \\
& \operatorname{Im}(\mathscr{N})_{1,3}=\frac{s_{2}\left(t_{1}^{2}+t_{2}^{2}-u_{1}^{2}-u_{2}^{2}\right)\left(2 t_{2} u_{2}-x_{2}^{2}\right)-\left(u_{2}\left(t_{1}^{2}+t_{2}^{2}-u_{1}^{2}+u_{2}^{2}\right)-t_{2} x_{2}^{2}\right) y_{2}^{2}}{\left(-2 t_{2} u_{2}+x_{2}^{2}\right)^{2}} \\
& \operatorname{Im}(\mathscr{N})_{1,4}=-\frac{\sqrt{2} s_{2}\left(\left(t_{1}+u_{1}\right) x_{1}+\left(t_{2}+u_{2}\right) x_{2}\right)}{-2 t_{2} u_{2}+x_{2}{ }^{2}}+
\end{aligned}
$$




$$
\begin{aligned}
& \frac{\left(-2\left(t_{1}+u_{1}\right) u_{2} x_{1}+2 u_{2}^{2} x_{2}+x_{2}^{3}\right) y_{2}^{2}}{\sqrt{2}\left(-2 t_{2} u_{2}+x_{2}^{2}\right)^{2}} \\
& \operatorname{Im}(\mathscr{N})_{1,5}=\frac{\sqrt{2}\left(2 u_{2}^{2}+x_{2}^{2}\right) y_{2}}{4 t_{2} u_{2}-2 x_{2}^{2}} \\
& \operatorname{Im}(\mathscr{N})_{2,2}=\frac{s_{2}\left(4+4 t_{1}{ }^{2}\left(u_{1}{ }^{2}+u_{2}{ }^{2}\right)+4 t_{2}{ }^{2}\left(u_{1}{ }^{2}+u_{2}{ }^{2}\right)-4 x_{1}{ }^{2}+x_{1}{ }^{4}+2 x_{1}{ }^{2} x_{2}{ }^{2}+x_{2}{ }^{4}\right)}{8 t_{2} u_{2}-4 x_{2}{ }^{2}}+ \\
& -\frac{s_{2}\left(t_{1}\left(2 u_{2} x_{1} x_{2}+u_{1}\left(-2+x_{1}^{2}-x_{2}^{2}\right)\right)+t_{2}\left(2 u_{1} x_{1} x_{2}+u_{2}\left(-x_{1}^{2}+x_{2}^{2}\right)\right)\right)}{2 t_{2} u_{2}-x_{2}^{2}}+ \\
& \frac{y_{1}\left(2 t_{2} u_{2}^{2} y_{1}-4 t_{1} u_{2}^{2} y_{2}-2 u_{1} x_{2}^{2} y_{2}+u_{2} x_{2}\left(-\left(x_{2} y_{1}\right)+4 x_{1} y_{2}\right)\right)}{4 t_{2} u_{2}-2 x_{2}^{2}}+ \\
& \frac{-u_{2}\left(4 t_{1}^{2}\left(u_{1}-u_{2}\right)\left(u_{1}+u_{2}\right)+4 t_{2}^{2}\left(u_{1}^{2}+u_{2}^{2}\right)-\left(2+4 t_{1} u_{1}-x_{1}^{2}\right)\left(-2+x_{1}^{2}\right)\right) y_{2}^{2}}{4\left(-2 t_{2} u_{2}+x_{2}^{2}\right)^{2}}+ \\
& \frac{-x_{2}\left(8 t_{1} u_{2}^{2} x_{1}-4\left(t_{2}\left(u_{1}^{2}+u_{2}^{2}\right)+u_{2}\left(t_{1} u_{1}+x_{1}^{2}\right)\right) x_{2}+4 u_{1} x_{1} x_{2}^{2}+u_{2} x_{2}{ }^{3}\right) y_{2}^{2}}{4\left(-2 t_{2} u_{2}+x_{2}^{2}\right)^{2}} \\
& \operatorname{Im}(\mathscr{N})_{2,3}=-\frac{s_{2}\left(2 t_{1}{ }^{2} u_{1}+2\left(-t_{2}+u_{2}\right) x_{1} x_{2}+u_{1}\left(-2+2 t_{2}{ }^{2}+x_{1}{ }^{2}-x_{2}{ }^{2}\right)+t_{1}\left(2-2 u_{1}{ }^{2}-2 u_{2}{ }^{2}-x_{1}{ }^{2}+x_{2}{ }^{2}\right)\right)}{4 t_{2} u_{2}-2 x_{2}{ }^{2}}+ \\
& \frac{u_{2}\left(2 t_{2}^{2} u_{1}+2 t_{1} u_{2}^{2}+\left(t_{1}-u_{1}\right)\left(2+2 t_{1} u_{1}-x_{1}^{2}-x_{2}^{2}\right)\right) y_{2}^{2}}{2\left(-2 t_{2} u_{2}+x_{2}^{2}\right)^{2}}+ \\
& \frac{-\left(y_{2}\left(x_{2}\left(-2 u_{2}^{2}+x_{2}^{2}\right)\left(x_{2} y_{1}-x_{1} y_{2}\right)+2 t_{2}\left(2 u_{2}^{3} y_{1}-u_{2} x_{2}^{2} y_{1}+u_{1} x_{2}^{2} y_{2}\right)\right)\right)}{2\left(-2 t_{2} u_{2}+x_{2}^{2}\right)^{2}} \\
& \operatorname{Im}(\mathscr{N})_{2,4}=\frac{s_{2}\left(x_{1}^{3}-2\left(t_{2} u_{1}+t_{1} u_{2}\right) x_{2}+x_{1}\left(-2-2 t_{1} u_{1}+2 t_{2} u_{2}+x_{2}^{2}\right)\right)}{\sqrt{2}\left(2 t_{2} u_{2}-x_{2}^{2}\right)}+ \\
& -\frac{\sqrt{2} u_{2} x_{2} y_{2}\left(-2 t_{2} u_{2} y_{1}+x_{2}^{2} y_{1}+t_{1} u_{2} y_{2}\right)}{\left(-2 t_{2} u_{2}+x_{2}^{2}\right)^{2}}+ \\
& -\frac{\left(u_{1} x_{2}^{3}+u_{2} x_{1}\left(-2-2 t_{1} u_{1}+x_{1}^{2}-2 x_{2}^{2}\right)\right) y_{2}^{2}}{\sqrt{2}\left(-2 t_{2} u_{2}+x_{2}^{2}\right)^{2}} \\
& \operatorname{Im}(\mathscr{N})_{2,5}=\frac{\sqrt{2}\left(2 t_{2} u_{2}^{2} y_{1}-2 t_{1} u_{2}^{2} y_{2}-u_{1} x_{2}^{2} y_{2}+u_{2} x_{2}\left(-\left(x_{2} y_{1}\right)+2 x_{1} y_{2}\right)\right)}{4 t_{2} u_{2}-2 x_{2}^{2}} \\
& \operatorname{Im}(\mathscr{N})_{3,3}=\frac{s_{2}\left(t_{1}{ }^{2}+t_{2}{ }^{2}-2 t_{1} u_{1}+u_{1}{ }^{2}+u_{2}{ }^{2}-x_{2}{ }^{2}\right)}{2 t_{2} u_{2}-x_{2}{ }^{2}}+ \\
& -\frac{\left(u_{2}\left(\left(t_{1}-u_{1}\right)^{2}+\left(t_{2}-u_{2}\right)\left(t_{2}+u_{2}\right)\right)+\left(-t_{2}+u_{2}\right) x_{2}^{2}\right) y_{2}{ }^{2}}{\left(-2 t_{2} u_{2}+x_{2}{ }^{2}\right)^{2}} \\
& \operatorname{Im}(\mathscr{N})_{3,4}=\frac{\sqrt{2} s_{2}\left(t_{1} x_{1}-u_{1} x_{1}+\left(t_{2}-u_{2}\right) x_{2}\right)}{2 t_{2} u_{2}-x_{2}^{2}}+ \\
& \frac{\left(-2 t_{1} u_{2} x_{1}+2 u_{1} u_{2} x_{1}-2 u_{2}^{2} x_{2}+x_{2}^{3}\right) y_{2}^{2}}{\sqrt{2}\left(-2 t_{2} u_{2}+x_{2}^{2}\right)^{2}} \\
& \operatorname{Im}(\mathscr{N})_{3,5}=\frac{\sqrt{2}\left(-2 u_{2}^{2}+x_{2}^{2}\right) y_{2}}{4 t_{2} u_{2}-2 x_{2}^{2}} \\
& \operatorname{Im}(\mathscr{N})_{4,4}=\frac{s_{2}\left(2 t_{2} u_{2}-x_{2}^{2}\right)\left(2 t_{2} u_{2}+2 x_{1}^{2}+x_{2}^{2}\right)+2 u_{2}\left(-x_{1}+x_{2}\right)\left(x_{1}+x_{2}\right) y_{2}{ }^{2}}{\left(-2 t_{2} u_{2}+x_{2}^{2}\right)^{2}} \\
& \operatorname{Im}(\mathscr{N})_{4,5}=\frac{2 u_{2} x_{2} y_{2}}{2 t_{2} u_{2}-x_{2}{ }^{2}} \\
& \operatorname{Im}(\mathscr{N})_{5,5}=u_{2}
\end{aligned}
$$

\section{References}

[1] G. Pradisi and A. Sagnotti, Phys. Lett. B216, 59 (1989); A. Sagnotti, Phys. Rept. 184, 167 (1989). 
[2] J. Polchinski and Y. Cai, Nucl. Phys. B296, 91 (1988); J. Dai, R. G. Leigh and J. Polchinski, Mod. Phys. Lett. A4, 2073 (1989).

[3] P. K. Tripathy and S. P. Trivedi, JHEP 0303, 028 (2003).

[4] L. Andrianopoli, R. D'Auria, S. Ferrara and M. A. Lledo, JHEP 0303, 044 (2003).

[5] T. R. Taylor and C. Vafa, Phys. Lett. B474, 130 (2000).

[6] P. Mayr, Nucl. Phys. B593, 99 (2001); P. Mayr, JHEP 0011, 013 (2000).

[7] G. Curio, A. Klemm, D. Lust and S. Theisen, Nucl. Phys. B609, 3 (2001).

[8] M. Berg, M. Haack and B. Kors, Nucl. Phys. B669, 3 (2003).

[9] J. Louis and A. Micu, Nucl. Phys. B635, 395 (2002).

[10] G. Curio, A. Klemm, B. Kors and D. Lust, Nucl. Phys. B620, 237 (2002).

[11] G. Dall'Agata, JHEP 0111, 005 (2001).

[12] G. L. Cardoso, G. Curio, G. Dall'Agata, D. Lust, P. Manousselis and G. Zoupanos, Nucl. Phys. B652, 5 (2003).

G. L. Cardoso, G. Curio, G. Dall'Agata and D. Lust, JHEP 0310, 004 (2003) .

[13] K. Becker, M. Becker, K. Dasgupta and P. S. Green, JHEP 0304, 007 (2003).

[14] S. Kachru, R. Kallosh, A. Linde and S. P. Trivedi, Phys. Rev. D68, 046005 (2003).

[15] J. P. Hsu, R. Kallosh and S. Prokushkin, JCAP 0312, 009 (2003).

[16] K. Dasgupta, C. Herdeiro, S. Hirano and R. Kallosh, Phys. Rev. D65, 126002 (2002).

[17] S. Kachru, R. Kallosh, A. Linde, J. Maldacena, L. McAllister and S. P. Trivedi, "Towards inflation in string theory", JCAP 0310, 013 (2003).

[18] C. P. Burgess, R. Kallosh and F. Quevedo, JHEP 0310056 (2003).

[19] F. Koyama, Y. Tachikawa and T. Watari, Phys. Rev. D69, 106001 (2004).

[20] M. K. Gaillard and B. Zumino, Nucl. Phys. B193, 221 (1981).

[21] C. Angelantonj, R. D'Auria, S. Ferrara and M. Trigiante, Phys. Lett. B 583, 331 (2004).

[22] B. de Wit and A. Van Proeyen, Commun. Math. Phys. 149, 307-334 (1992). 
[23] R. D'Auria, S. Ferrara, F. Gargiulo, M. Trigiante and S. Vaula, JHEP 0306, 045 (2003).

[24] A. Salam and E. Sezgin, (Ed.), "Supergravities In Diverse Dimensions. Vol. 1, 2", Amsterdam, Netherlands: North-Holland (1989) 1499 p.

[25] L. Andrianopoli, R. D'Auria and S. Ferrara, Int. J. Mod. Phys. A13, 431 (1998).

[26] C. Angelantonj, S. Ferrara and M. Trigiante, JHEP 0310, 015 (2003); Phys. Lett. B582, 263 (2004).

[27] J. Polchinski and A. Strominger, Phys. Lett. B388 736 (1996).

[28] L. Andrianopoli, M. Bertolini, A. Ceresole, R. D'Auria, S. Ferrara, P. Fré and T. Magri, Jour. Geom and Phys. Vol. 23, 111 (1997).

[29] A. Ceresole, R. D'Auria, S. Ferrara and A. Van Proeyen, Nucl. Phys. B444, 92 (1995).

[30] A. R. Frey and J. Polchinski, Phys. Rev. D65, 126009 (2002).

[31] S. Kachru, M. Schulz and S. Trivedi, JHEP 0310, 007 (2003).

[32] S. Cecotti, L. Girardello and M. Porrati, Phys. Lett. B145, 61 (1984).

[33] E. Cremmer, S. Ferrara, C. Kounnas and D. V. Nanopoulos, Phys. Lett. B133, 61 (1983).

[34] J. R. Ellis, A. B. Lahanas, D. V. Nanopoulos and K. Tamvakis, Phys. Lett. B134, 429 (1984); J. R. Ellis, C. Kounnas and D. V. Nanopoulos, Nucl. Phys. B247, 373 (1984); A. B. Lahanas and D. V. Nanopoulos, Phys. Rept. 145, 1 (1987).

[35] S. Ferrara and M. Porrati, Phys. Lett. B545, 411 (2002).

[36] S. Ferrara, R. Minasian and A. Sagnotti, Nucl. Phys. B474, 323 (1996).

[37] I. Antoniadis, C. Bachas, C. Fabre, H. Partouche and T. R. Taylor, Nucl. Phys. B489, 160 (1997).

[38] D. V. Alekseevskii, Izv. Akad. Nauk SSSR Ser. Mat. 9, 315-362 (1975); Math. USSR Izvesstija, 9, 297-339 (1975).

[39] S. Cecotti, Commun. Math. Phys. 124, 23 (1989).

[40] E. Halyo, Phys. Lett. B387, 43 (1996). 
[41] R. Kallosh, "N = 2 supersymmetry and de Sitter space," arXiv:hep-th/0109168.

[42] A. D. Linde, Phys. Rev. D49, 748 (1994). 